\title{
Structure of Spinel-Type Phases in the $\mathrm{ZnO}-\mathrm{Mn}_{\mathrm{x}} \mathrm{O}_{\mathrm{y}}$ System
}

\author{
Marco Peiteado, ${ }^{\dagger, \ddagger, \S}$ Alojz Kodre, ${ }^{\boldsymbol{\eta}, \|}$ Iztok Arčon, ${ }^{\| \dagger \dagger}$ Amador C. Caballero, ${ }^{\S}$ and Darko Makovec ${ }^{\ddagger}$ \\ ${ }^{\ddagger}$ Synthesis of Materials Department, Jožef Stefan Institute, 1000 Ljubljana, Slovenia \\ ${ }^{\S}$ Department of Electroceramics, Instituto de Cerámica y Vidrio, CSIC, 28049 Madrid, Spain \\ ${ }^{\top}$ Faculty of Mathematics and Physics, University of Ljubljana, 1000 Ljubljana, Slovenia \\ "Jožef Stefan Institute, 1000 Ljubljana, Slovenia \\ ${ }^{\dagger}$ University of Nova Gorica, 5000 Nova Gorica, Slovenia
}

\begin{abstract}
The microstructural development of the $\mathrm{Zn}-\mathrm{Mn}-\mathrm{O}$ system in air atmosphere is governed by the formation and thermal evolution of different $\mathrm{Mn}_{3-x} \mathrm{Zn}_{x} \mathrm{O}_{4}$ spinel-type phases, whose degree of distortion varies as a function of composition and temperature. By means of X-ray powder diffractometry and X-ray absorption spectroscopy, the structure of the observed cubic $\mathrm{C}$ and tetragonal $\mathrm{T} 1$ and $\mathrm{T} 2$ spinels is analyzed in the present contribution. $X$-ray absorption near-edge structure reveals a decrease of the average Mn valence with the increasing calcination temperature. Local structures of the spinel phases have been studied using simultaneous refinement of the X-ray absorption fine structure of both metals. The analysis has found deformations of the $\mathrm{MnO}_{6}$ octahedra already in the $\mathrm{C}$ phase. With the orientation of these random deformations to a common $c$-axis, the cubic $\mathrm{C}$ phase transforms into the tetragonal $\mathrm{T} 2$ phase. With increasing temperature, the tetragonal character of the $\mathrm{T} 2$ phase becomes ever more pronounced as the content of $\mathrm{Mn}^{3+}$ increases and the value of $\boldsymbol{x}$ decreases. In $\mathrm{C}$ and $\mathrm{T} 2$ phases, Mn only occupies octahedral sites of the spinel structure, whereas in the $\mathrm{T} 1$ phase, a small degree of inversion was observed.
\end{abstract}

\section{Introduction}

Z INC manganites of nominal composition $\mathrm{Mn}_{3-x} \mathrm{Zn}_{x} \mathrm{O}_{4}$ find technological application as negative temperature coefficient (NTC) thermistors. ${ }^{1}$ Because of their spinel-like structure with a large capability to form stable and variable solid solutions, these oxides are also used as enhanced catalysts in the process of removal gaseous pollutants from atmosphere ${ }^{2,3}$ and, when coloring cations are involved, they also find application in the field of ceramic pigments. ${ }^{4}$ But moreover, in the past decade, a growing interest in the $\mathrm{Mn}-\mathrm{Zn}-\mathrm{O}$ system has emerged because of its promising application as a dilute magnetic semiconductor for spintronics devices. ${ }^{5,6}$ Although most of the research in this field is directed to understand the mechanism responsible for the room-temperature magnetic behavior of this candidate solid solution, the solid-state community is also putting efforts in describing the microstructural and structural features involved in this pseudo-binary system. The first studies on the reactivity between $\mathrm{ZnO}$ and manganese oxides date back to the 1960's and

T. Vanderah—contributing editor

Manuscript No. 26173. Received April 23, 2009; approved September 14, 2009.

This work was supported by Slovenian Research Agency research programs P2-0089 and P1-0112, by DESY and the European Community under Contract RII3-CT-2004506008 (IA-SFS), and by CICYT MAT 2007-65857 and CICYT MAT 2007-66845-C02-01 projects.

${ }^{\dagger}$ Author to whom correspondence should be addressed. e-mail: mpeiteado@icv.csic.es suggest the existence of several unidentified spinel-like structures, mixed oxides that can be ascribed to the $\mathrm{Zn}_{x} \mathrm{Mn}_{3-x} \mathrm{O}_{4+y}$ system. ${ }^{7,8}$ The proposed scenario has been recently confirmed and updated with several works that demonstrate that whenever an equilibrium situation is achieved, the formation and thermal evolution of different $\mathrm{Mn}_{3-x} \mathrm{Zn}_{x} \mathrm{O}_{4}$ spinel phases governs the microstructural development of the $\mathrm{Zn}-\mathrm{Mn}-\mathrm{O}$ system in air atmosphere. ${ }^{1,9-11}$ The fact is that at room temperature, a manganite can have a tetragonal as well as a cubic structure, and this finally depends on the concentration of Mn (III) ions in the octahedral sites of the spinel lattice. Mn (III) has a $d^{4}$ electronic configuration, which makes it a strongly active Jahn-Teller ion. As the doublet states cannot be degenerated in the crystal field, this means that in the case of orbital degeneracy, an octahedron of ligands around the Mn (III) is distorted in such a way that $c / a>1$. Above a certain concentration of these ions, the cooperation of many distortions in the crystal leads to the distortion of the structure as a whole. Accordingly, the increase of the Mn (III) ions concentration in the octahedral sites results in the order (cubic) - disorder (tetragonal) transition. In the particular case of $\mathrm{Mn}_{3-x} \mathrm{Zn}_{x} \mathrm{O}_{4}$, zinc manganites, it has been shown that the concentration of Mn (III) ions in the octahedral sites varies with two parameters, composition and temperature. ${ }^{1,8,9,12}$ Actually in a previous report, we have analyzed the thermal evolution of the $\mathrm{Zn}-\mathrm{Mn}-\mathrm{O}$ system in air atmosphere by means of $\mathrm{X}$-ray powder diffraction (XRD), transmission electron microscope with energy-dispersive X-ray spectroscopy (TEM-EDS), and electron energy loss spectroscopy (EELS) analyses. ${ }^{13}$ The results obtained suggested that this evolution certainly involves the formation of several $\mathrm{Mn}_{3-x} \mathrm{Zn}_{x} \mathrm{O}_{4}$ spinel-type phases, and different fields of stability were defined, which are summarized in the scheme of Fig. 1. As observed, at low temperatures, a cubic spinel $\mathrm{C}$ is stable with the composition defined with $x=1.67$ and the octahedral sites occupied by a mixture of $\mathrm{Mn}^{3+}, \mathrm{Mn}^{4+}$, and $\mathrm{Zn}^{2+}$ ions: $\left(\mathrm{Zn}^{2+}\right)\left[\mathrm{Zn}_{x-1}^{2+} \mathrm{Mn}_{x-1}^{4+} \mathrm{Mn}_{4-2 x}^{3+}\right] \mathrm{O}_{4}$ (this formula was taken from Driessen and Rieck ${ }^{8}$ ). When the amount of zinc is decreased (lower $x$ ), the overall amount of $\mathrm{Mn}^{3+}$ in the octahedral sites is thus increased; hence, the cubic phase coexists with a distorted tetragonal spinel T1. For $x=1$, the only stable phase is the distorted T1 structure, with all manganese in its oxidation state III: tetragonal $\left(\mathrm{Zn}^{2+}\right)\left[\mathrm{Mn}_{2}^{3+}\right] \mathrm{O}_{4}$. This $\mathrm{T} 1$ phase remains stable for the whole temperature range. However, the cubic phase undergoes a structural transformation above $700^{\circ} \mathrm{C}$, which leads to the formation of a second tetragonal spinel $\mathrm{T} 2$. The process was attributed to the continuing reduction of $\mathrm{Mn}$ (IV) to $\mathrm{Mn}$ (III). With increasing the temperature, the tetragonal character of the $\mathrm{T} 2$ phase becomes even more pronounced as the content of $\mathrm{Mn}^{3+}$ increases and the value of $x$ decreases, until finally the T2 phase leads to the higher distortion of the T1 structure. 


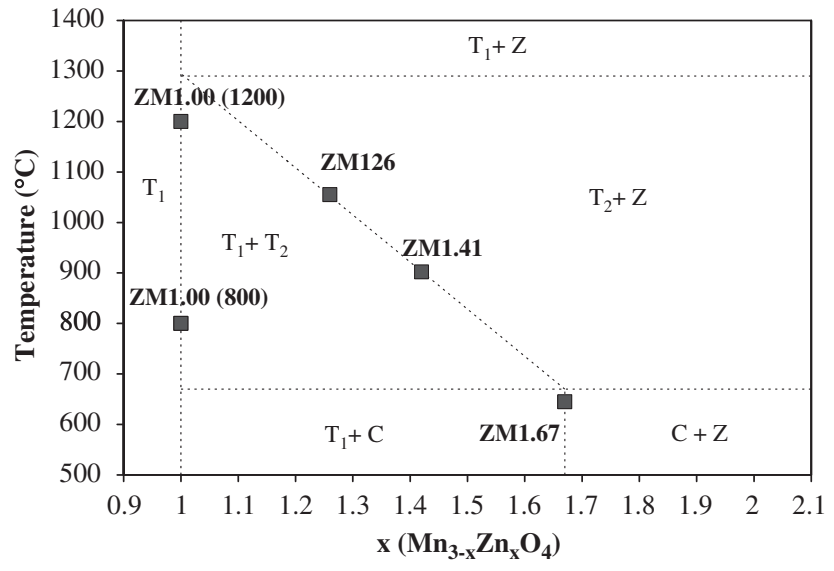

Fig. 1. Stability regions in the $\mathrm{Zn}-\mathrm{Mn}-\mathrm{O}$ system in air as a function of temperature and composition, as determined in Peiteado et al. ${ }^{13}$ Black dots correspond to the samples analyzed in the present study to determine the crystal structure of the spinel phases observed. T1, tetragonal $\mathrm{ZnMn}_{2} \mathrm{O}_{4}$ spinel; $\mathrm{C}$, cubic spinel; $\mathrm{T}$ 2, tetragonal spinel; $\mathrm{Z}$, hexagonal $\mathrm{ZnO}$.

With all these results, the mechanisms behind these transformations were established. However, the crystal chemistry of the phases involved has not been clarified yet. By means of mainly XRD experiments, several authors have suggested different cationic distributions for these $\mathrm{Zn}-\mathrm{Mn}$ spinels, ${ }_{1,8,9,14}$ but a clear picture of their structural parameters remains to be described. In the present contribution, we meet this goal using a combination of classical macroscopic methods and extended X-ray absorption fine structure (EXAFS) and X-ray absorption near-edge structure (XANES) spectroscopy techniques.

\section{Experimental Procedure}

Polycrystalline samples with the nominal composition $\mathrm{Mn}_{3-x}$ $\mathrm{Zn}_{x} \mathrm{O}_{4}$ with $1 \leq x \leq 2$ were obtained via a single-source crystalline oxalate precursor, which ensures random atomic-scale mixing of the ions involved. ${ }^{15}$ The precursor was obtained by a room-temperature coprecipitation with oxalic acid of aqueous solutions of zinc and manganese acetates, $\mathrm{Zn}\left(\mathrm{CH}_{3} \mathrm{COO}\right)_{2} \cdot 2 \mathrm{H}_{2} \mathrm{O}$ and $\mathrm{Mn}\left(\mathrm{CH}_{3} \mathrm{COO}\right)_{2} \cdot 4 \mathrm{H}_{2} \mathrm{O}$. Reagent-grade raw materials (SigmaAldrich, Taufkirchen, Germany) were used in all cases. The precipitated oxalate was calcined at $400^{\circ} \mathrm{C}$ to ensure a clean decomposition into the corresponding oxides. More details about the synthesis method are described elsewhere. ${ }^{16}$ The calcined powders were pressed into pellets and heated at temperatures ranging from $650^{\circ}$ to $1300^{\circ} \mathrm{C}$. The final temperatures and compositions were chosen to result in the single-phase spinels. Furthermore, to avoid any parasitic or secondary reaction, the pellets were cautiously introduced into the furnace once the heating temperature was reached. At this temperature, they were subjected to a $100 \mathrm{~h}$ dwell time and finally, they were rapidly quenched in liquid nitrogen to freeze in the high-temperature state. The compositions and firing temperatures for the analyzed samples are listed in Table I.
The composition of the samples after the precipitation process was verified by inductively coupled plasma atomic emission spectroscopy (ICP-AES) on a Atomscan 25 Spectrometer (Thermo Jarrell Ash Corp., Franklin, MA). The sintered pellets were ground up and characterized by means of XRD. For the XRD profile analyses, step-scanned patterns were collected between $15^{\circ}$ and $65^{\circ}$ on an AXS Endeavor 4 diffractometer (Bruker, Karlsruhe, Germany) in steps of $0.02^{\circ}$ and a counting time of $12 \mathrm{~s}$ per step. The lattice parameters were estimated by fitting the powder XRD profiles, using the nonlinear least-squares method. The single-phase nature, homogeneity, and compositions of the samples were also verified using TEM-EDS, following the methodology explained in Peiteado et al. ${ }^{13}$

The local structure in the neighborhood of the constituent metal cations in the samples was studied by X-ray absorption spectroscopy. For XAS analysis, the powder samples were mixed with boron nitride and pressed into thin homogeneous pellets. The absorption thickness of a pellet was about 1.5 above the Mn and $\mathrm{Zn} \mathrm{K}$-edge absorption edge. EXAFS spectra at the K-edges of the metals were measured in a standard transmission mode at the C station of HASYLAB synchrotron facility, DESY (Hamburg, Germany). A Si(111) double-crystal monochromator was used with $1 \mathrm{eV}$ resolution at $7 \mathrm{keV}$. The harmonics were effectively eliminated by detuning the monochromator crystal using a stabilization feedback control. The three ionization detectors were filled with $1000 \mathrm{mbar}_{2}, 180 \mathrm{mbar} \mathrm{Ar}$, and $320 \mathrm{mbar} \mathrm{Ar}$ for Mn EXAFS, and with 140 mbar Ar, 900 mbar Ar, and 900 mbar Ar for Zn EXAFS. Sample pellets were placed between the first two detectors. The exact energy calibration with a precision of $0.05 \mathrm{eV}$ was established with a simultaneous absorption measurement on the corresponding ( $\mathrm{Zn}$ or $\mathrm{Mn}$ ) metal foil placed between the second and the third ionization detector.

The absorption spectra were measured within the interval from 250 to $1000 \mathrm{eV}$ relative to the $\mathrm{K}$-edge of the constituent metal. In the XANES region, equidistant energy steps of $0.25 \mathrm{eV}$ were used for a precise determination of the edge shape and position, while for the EXAFS region, equidistant k-steps $\left(\Delta k \approx 0.03 \AA^{-1}\right)$ were adopted with an integration time of 2 $\mathrm{s} /$ step. The position of $\mathrm{Zn}$ edge was, within experimental error, the same for all samples, while the position of Mn edge varied systematically, calling for XANES measurement of a number of auxiliary samples of $\mathrm{Mn}$ compounds with a well-defined $\mathrm{Mn}$ valence ( $\mathrm{MnO}$ (II), $\mathrm{Mn}_{3} \mathrm{O}_{4}$ (II, III), $\mathrm{Mn}_{2} \mathrm{O}_{3}$ (III), and $\mathrm{MnO}_{2}$ (IV)).

The spectra were analyzed with the IFEFFIT code. ${ }^{17,18}$ The FEFF model of the EXAFS signal at the two metal K-edges was built from the crystallographic data on the MZT spinel. ${ }^{19}$ Following the argumentation in the study of Calvin, ${ }^{20}$ a comprehensive FEFF calculation was adopted with a simultaneous relaxation of the two spectra, exploiting connections and restraints between the parameters of the EXAFS models of the constituent elements. The advantage of this approach, with regard to the separate fitting of individual EXAFS signals, lies in better statistical reliability of determined structural parameters.

\section{Results and Discussion}

Table I lists the lattice parameters of the analyzed samples obtained from the XRD spectra shown in Fig. 2. XRD shows a

Table I. Nominal Composition (Expressed as $x$ in $\mathrm{Mn}_{3-x} \mathrm{Zn}_{x} \mathrm{O}_{4}$ Formula), Firing Temperature, and Lattice Parameters of the Samples Analyzed in this Study

\begin{tabular}{|c|c|c|c|c|c|}
\hline Sample & $\begin{array}{c}\text { Composition } \\
\left(x \text { in } \mathrm{Mn}_{3-x} \mathrm{Zn}_{x} \mathrm{O}_{4}\right)\end{array}$ & Temperature $\left({ }^{\circ} \mathrm{C}\right)$ & Phase & \multicolumn{2}{|c|}{ Lattice parameters $(\AA)$} \\
\hline ZM1.67 & 1.67 & 650 & $\mathrm{C}$ & $8.3745(1)$ & 1 \\
\hline ZM1.26 & 1.26 & 1070 & $\mathrm{~T} 2$ & $5.7680(1)$ & $8.9709(2)$ \\
\hline ZM1.00 (800) & 1 & 800 & $\mathrm{~T} 1$ & $5.7194(1)$ & $9.2242(2)$ \\
\hline ZM1.00 (1200) & 1 & 1200 & $\mathrm{~T} 1$ & $5.7122(2)$ & $9.2221(4)$ \\
\hline
\end{tabular}




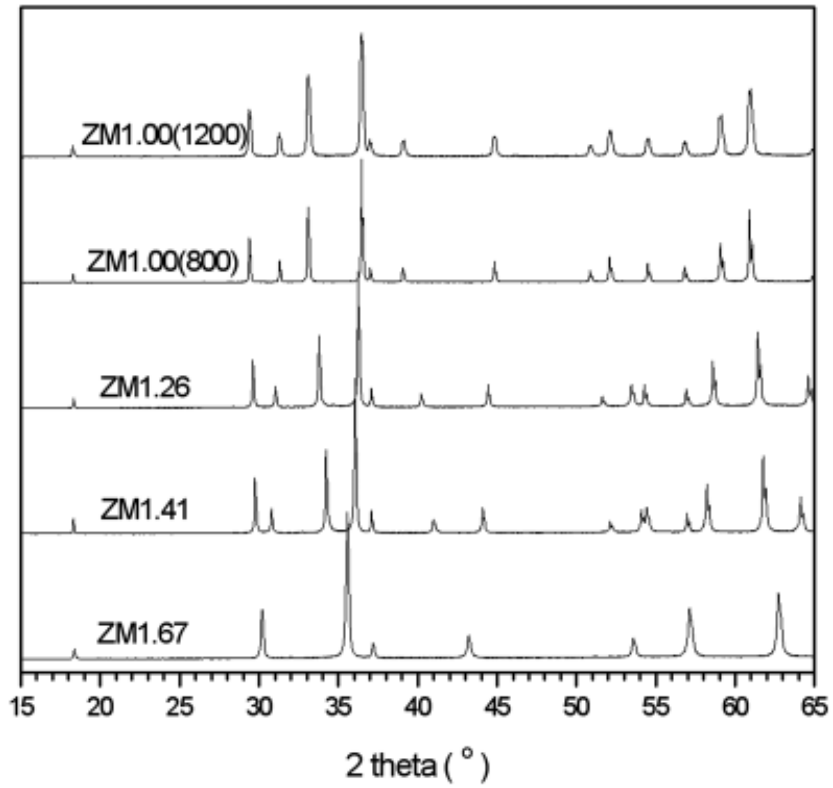

Fig. 2. X-ray powder diffraction spectra of the MnZn spinel samples.

single-phase nature of the samples and their structure that is in agreement with previous works ${ }^{13}$ : the sample $\mathrm{ZM} 1.67$ treated at $650^{\circ} \mathrm{C}$ contains the cubic $\mathrm{C}$ phase, the samples ZM1.41 and $\mathrm{ZM} 1.26$, respectively, treated at $900^{\circ}$ and $1070^{\circ} \mathrm{C}$, contain the tetragonal T2 phase, and the samples ZM1.00 contain the tetragonal $\mathrm{T} 1$ phase independent of the firing temperature.

The average oxidation number of $\mathrm{Mn}$ and $\mathrm{Zn}$ cations can be deduced from the energy shift of the absorption edge. A linear relation between the edge shift and the valence state has been established for atoms with the same type of ligands. ${ }^{21-23}$

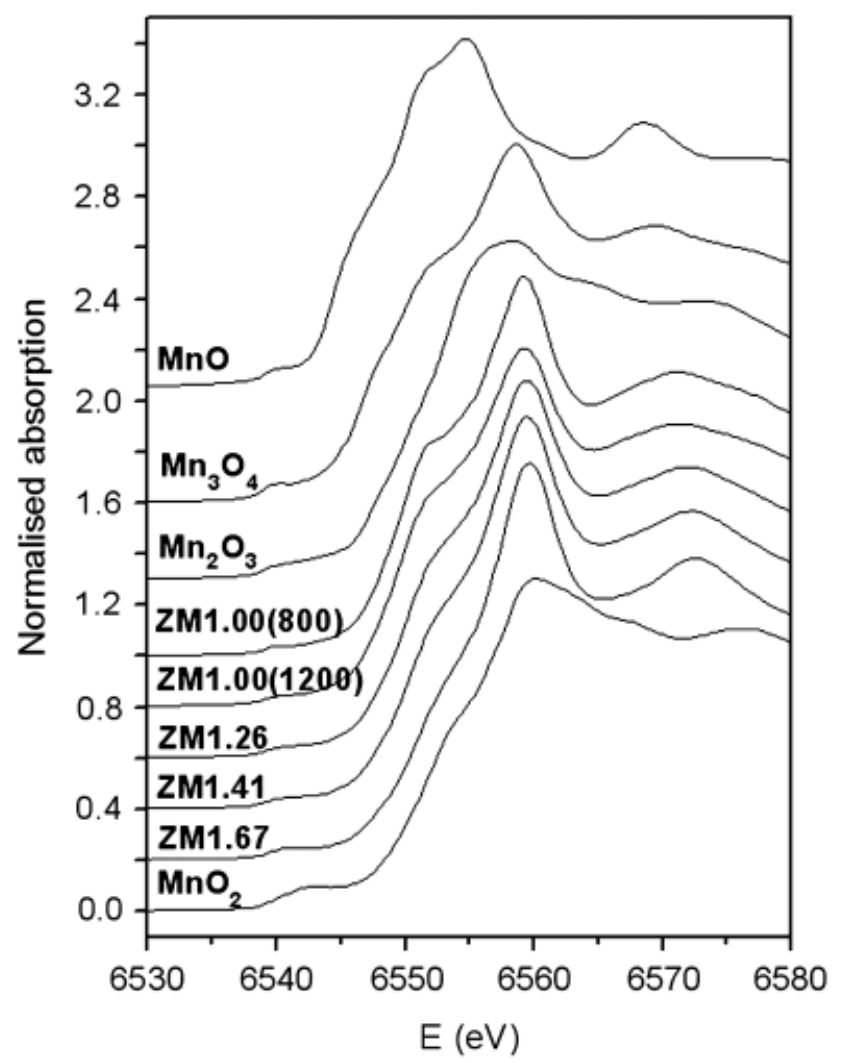

Fig. 3. Mn X-ray absorption near-edge structure spectra measured on the $\mathrm{MnZn}$ spinel samples and on $\mathrm{Mn}$ oxides: $\mathrm{MnO}, \mathrm{Mn}_{3} \mathrm{O}_{4}, \mathrm{Mn}_{2} \mathrm{O}_{3}$, $\mathrm{MnO}_{2}$.
$\mathrm{Zn}$ K-edge XANES spectra shows pure $\mathrm{Zn}$ (II) valence in all measured samples. In Fig. 3, Mn XANES profiles for the samples and some standard Mn oxides are presented. The edge shift clearly indicates that the average $\mathrm{Mn}$ valence state in the samples increases with an increasing value of $x$.

In addition, the variation in Mn edge profiles in Fig. 3 shows that the ligand environment in the $\mathrm{ZnMn}$ spinel samples differs somewhat from that in the reference oxides. The interpolation of sample valences from reference values may thus depend on the point in the edge profile, chosen for the comparison. This ambiguity is resolved by positioning the $\mathrm{Mn}$ valence in both ZM1.00 samples with standard spinel stoichiometry to $3+$, and using the rate of $3.5 \mathrm{eV}$ per unit valence change, derived from the reference data, to extrapolate the average Mn valence in the other three samples. The shifts are then defined from the position of the first inflection point in the edge profile. The accuracy of the derived values is conservatively estimated to $\pm 5 \%$. Table II shows the average valences obtained for these samples: apparently, they correspond closely to the nominal spinel formulas of the samples, suggesting no need for the formation of any extra charged point defects such as metal or oxygen vacancies. It is significant that although at low temperatures in air, the manganese oxidation state $4+$ is preferred, the average manganese valence of $3.5+$ in the sample ZM1.67, fired at the lowest temperature of $650^{\circ} \mathrm{C}$ already requires half of manganese in the oxidation state 3+. Moreover, as the value $x$ in the $\mathrm{Mn}_{3-x} \mathrm{Zn}_{x} \mathrm{O}_{4}$ formula decreases and progressively higher firing temperatures are applied, the average $\mathrm{Mn}$ valence decreases further.

The local structure of the MnZn spinel phases has been studied using EXAFS (Fig. 4). The Fourier transforms (FT) of the EXAFS spectra (Fig. 5) show large differences in positions and amplitudes of the peaks, pertaining to consecutive shells of neighbors. The differences stem from the different composition and structure of the samples, especially from their symmetry and from the occupation of two spinel lattice sites (tetrahedrally coordinated A sites and octahedrally coordinated B sites) by two cations. Generally, the ions in either site are surrounded by a shell of oxygen atoms at the distance of $\sim 2 \AA$-hence, the position of the first peak is rather steady, but the amplitude may vary depending on the distribution of the metal over the A and $\mathrm{B}$ sites, providing four and six oxygen neighbors, respectively. The effect of site occupation is more conspicuous in the second peak, due to the metal neighbors in either site. Its variable splitting is due to the large difference in site distances: $d(\mathrm{~A}-\mathrm{A})=3.75$ $\AA, d(\mathrm{~A}-\mathrm{B})=3.5 \AA, d(\mathrm{~B}-\mathrm{B})=3.0 \AA$, and coordination numbers $N(\mathrm{~A}-\mathrm{A})=4, N(\mathrm{~A}-\mathrm{B})=12, N(\mathrm{~B}-\mathrm{A})=6, N(\mathrm{~B}-\mathrm{B})=6$. This is also the basis for reliable determination of site occupation in quantitative analysis. ${ }^{20,24}$

The $a b$ initio model for quantitative analysis in the FEFF code $^{17,18}$ is built from crystallographic data for cubic spinel, ${ }^{19}$ where the real coordinates of the neighbors around the target atom are defined with only two parameters, the isotropic expansion coefficient and the oxygen parameter. For either metal, the model comprises two sets of scattering paths, one for each lattice site. Within the range of $3.75 \AA$, direct-scattering contributions of two shells of oxygen neighbors and two shells of metal neighbors are included, the contribution of multiple-scattering paths is negligible. The paths are described with standard EXAFS parameters, the Debye-Waller widths of the distribution of the interatomic distances, and the photoelectron zero energy correction of the two metals. The occupation probabilities of the two metals for the sites $\mathrm{A}$ and $\mathrm{B}\left(\mathrm{Mn}_{\mathrm{A}}, \mathrm{Mn}_{\mathrm{B}}, \mathrm{Zn}_{\mathrm{A}}\right.$, and $\mathrm{Zn}_{\mathrm{B}}$ ) can be reduced to a single parameter, taking into account the stoichiometric constraints.

This basic model does not provide a good fit to any of the five samples: a typical value of the $r$-factor, the quality-of-fit measure, is 0.15 . In the same way as in earlier studies, ${ }^{20,24}$ new degrees of freedom need to be introduced. Our goal is to find a sufficiently flexible model to describe the entire set of measured structures, but with as few additional parameters as possible, so that their structural function remains clear. In the opposite case, 
Table II. Mn Valence Determined from XANES Analysis and Structural Parameters from Modeling of the EXAFS Spectra: Zero

Energy for Mn (the Value for Zn is Kept Fixed), the Isotropic Expansion, and the Oxygen Parameter of the Basic Model, the Stoichiometric Ratios of $\mathrm{Zn}$ at $\mathrm{B}$ site and $\mathrm{Mn}$ at $\mathrm{A}$ site, the Tetragonal Deformation $\varepsilon$, the Debye-Waller Widths, and the Jahn-Teller correction $d_{\mathrm{MnO}}$

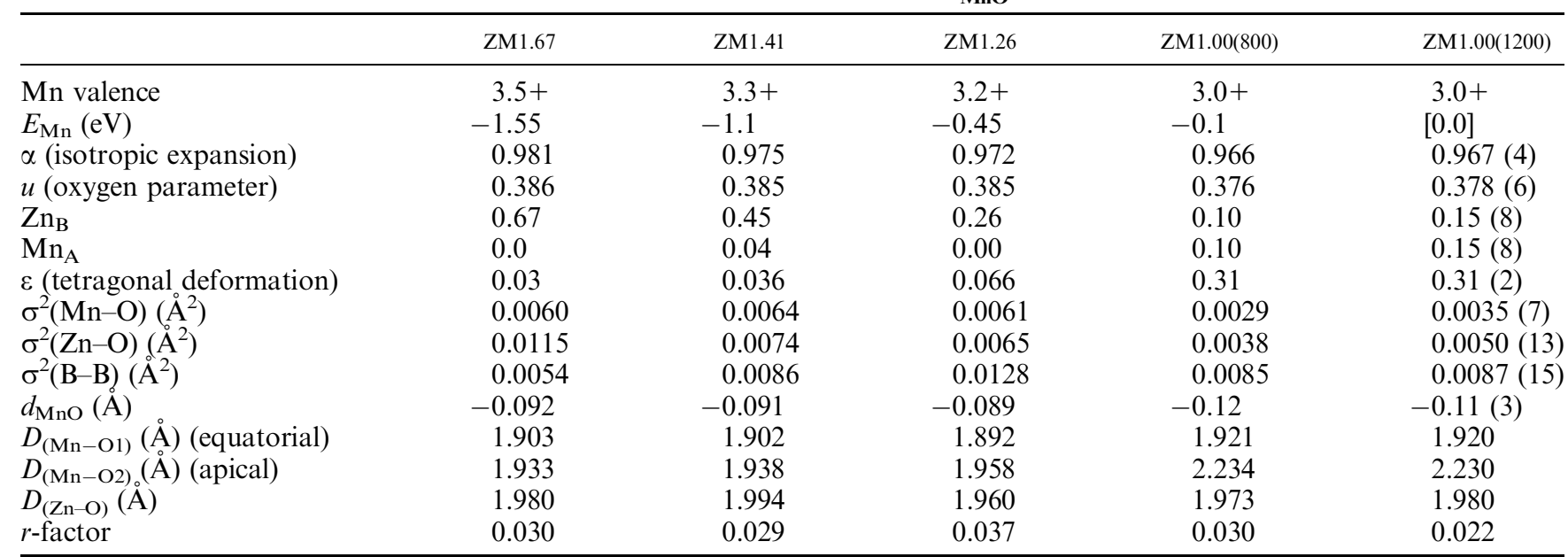

The combined model values for metal-oxygen distances D and the $r$-factors of the fits are given at the bottom. The amplitude reduction factors $\mathrm{S}^{2}$ are kept fixed at 0.75 and 0.90 for $\mathrm{Mn}$ and $\mathrm{Zn}$, respectively. Typical uncertainties of parameters in units of the last decimal place are given in parentheses in the last column. XANES; X-ray absorption near-edge structure; EXAFS, X-ray absorption fine structure.

a flood of parameters can provide perfect fits, but their values change almost randomly from sample to sample. The optimum in the complexity of the model is best found by trial and error: introducing new parameters with a clear physical meaning, and keeping those that bring an essential improvement to a majority of sample fits.

It turned out that all the extensions of the model-involved adaptation of the interatomic distances. The most salient feature of the measured $\mathrm{MnZn}$ spinels is their crystallographic variation, so that a deviation from cubic symmetry must be allowed. To describe the tetragonal deformation of the structure, the interatomic distances are expanded to the first order in the $\varepsilon=(c-a) / a$ ratio, and a best-fit value of $\varepsilon$ is determined for each sample. The unusually large value of Mn width parameters obtained in the basic model indicated split neighbors shells, conceivably through the Jahn-Teller effect, dividing the shell of six nearest oxygen neighbors into subshells of $4+2$ atoms, the latter at a distance increased by an additional parameter $d_{\mathrm{MnO}}$. Each of the new degrees of freedom effectively decreased the $r$-factor by one-half.

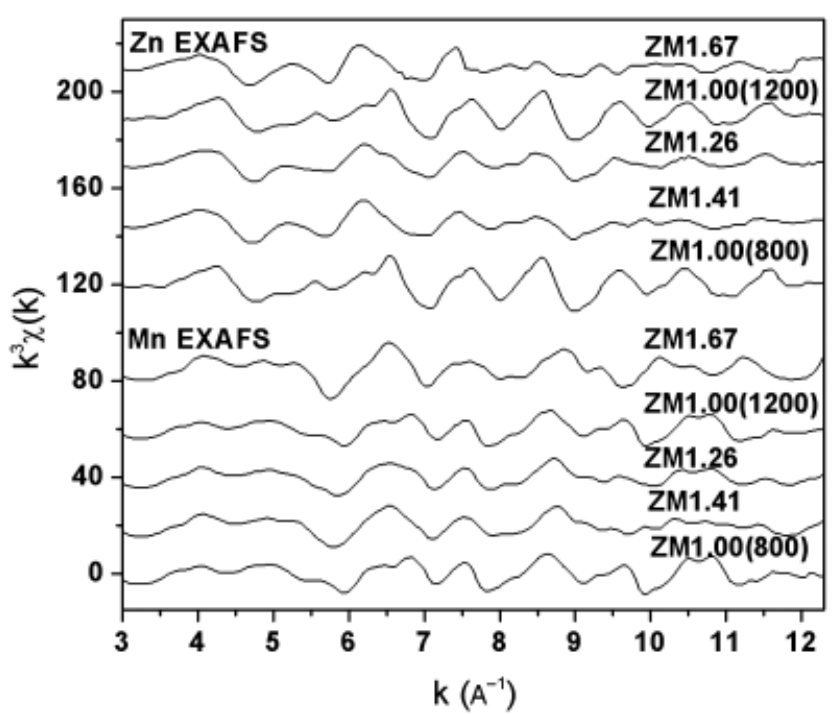

Fig. 4. The $\mathrm{k}^{3}$-weighted $\mathrm{Mn}$ and $\mathrm{Zn} \mathrm{X}$-ray absorption fine structure (EXAFS) spectra of the MnZn-spinel samples.
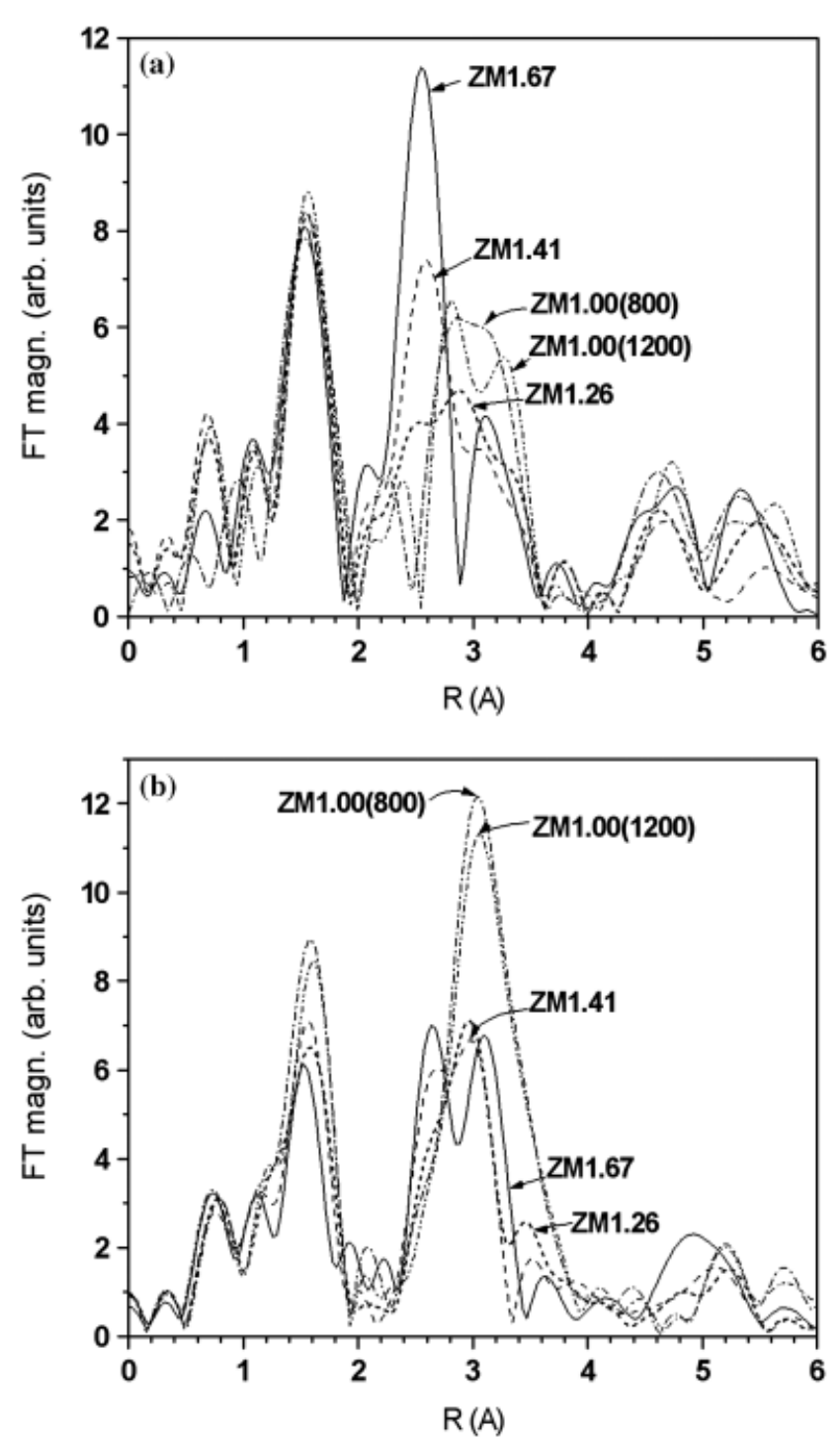

Fig. 5. Fourier transforms (FT) X-ray absorption fine structureS $\mathrm{k}^{3}$ weighted spectra from $\mathrm{Mn}$ (a) and $\mathrm{Zn}$ (b) K-edge in the MnZn-spinel samples. 

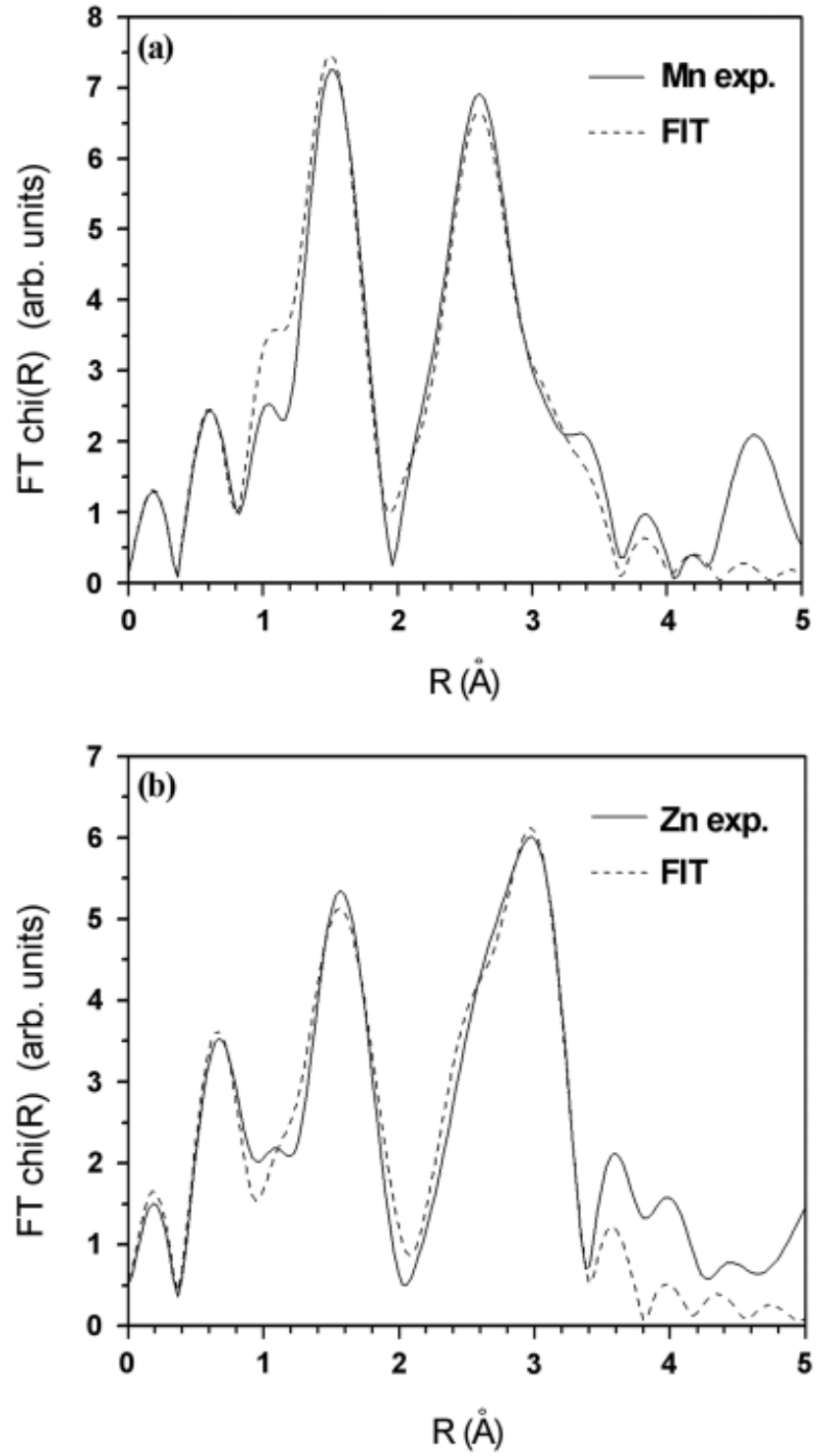

Fig. 6. Fourier transforms (FT) magnitude of the $\mathrm{k}^{3}$-weighted Mn (a) and $\mathrm{Zn}$ (b) X-ray absorption fine structure data (solid line) with the model (dashed) for sample ZM1.41. The background spinel coefficients are relaxed together with the model parameters to improve the fit of the first-neighbor peak.

The introduction of two additional deformation parameters can be rationalized as follows: the Jahn-Teller effect, which deforms the Mn octahedron, does not necessarily deform the longrange structure. In the $\mathrm{Zn}-\mathrm{Mn}-\mathrm{O}$ system, a cubic region exists, probably on account of isotropic orientations of the Jahn-Teller deformations of the Mn octahedra, randomly along any of the three axes. Only within certain thermodynamic conditions, upon the order-disorder transition, the octahedra are oriented to a common $c$-axis, and a tetragonal lattice is produced. More generally it means that the Jahn-Teller distance splitting and the splitting, introduced by the tetragonal deformation need not be the same-hence, the need for two independent parameters to describe the actual $\mathrm{Mn}-\mathrm{O}$ distances.

The best-fit structural parameters, obtained in a simultaneous fit in the k-range 3.6 $\AA^{-1}-12.8 \AA^{-1}$ for $\mathrm{Mn}$ and $4.4 \AA^{-1}-11.9$ $\AA^{-1}$ for Zn, are shown in Table II for all samples. In Fig. 6, the results of least-square relaxation of the EXAFS spectra of the sample ZM1.41 to the model is shown.

Apparently, some parameters follow the trend of the Mn valence: zero energy correction, $\mathrm{E}_{\mathrm{Mn}}$, the isotropic expansion $\alpha$, and the occupation parameters. In agreement with the strong preference of $\mathrm{Zn}$ ions for tetrahedral coordination, $\mathrm{Zn}$ enters the spinel $\mathrm{AB}_{2} \mathrm{O}_{4}$ structure preferentially at tetrahedral $\mathrm{A}$ sites, while $\mathrm{Mn}$ is situated at octahedral $\mathrm{B}$ sites. As evident from the table, the swapping of sites is mostly limited to the requirements of stoichiometry. Only ZM1.0 samples show some "spontaneous" swapping. Such swapping of $\mathrm{Zn}$ from tetrahedral to octahedral sites of the spinel structure is very limited in the bulk, but quite frequently observed in nanoparticles. ${ }^{24-26}$ The tetragonal parameter $\varepsilon$ distinctly shows the T1 phase while the transition from $\mathrm{C}$ to $\mathrm{T} 2$ remains moot: the arguments from the above discussion may apply. The same trend is observed in $d_{\mathrm{MnO}}$ and in the oxygen parameter $\mathrm{u}$. The metal-oxygen distances are, in effect, superposition of four contributions, the expansion, the change in the oxygen parameters, and the deformations $\varepsilon$ and $d_{\mathrm{MnO}}$. To make the results more transparent, the table is extended with the summary $\mathrm{Mn}-\mathrm{O}$ and $\mathrm{Zn}-\mathrm{O}$ distances. As observed, $D_{(\mathrm{Zn}-\mathrm{O})}$ and the distance of equatorial $\mathrm{Mn}-\mathrm{O}$ bonds $D_{(\mathrm{Mn}-\mathrm{O} 1)}$ remain almost invariable, but the distance of two polar oxygens $D_{(\mathrm{Mn}-\mathrm{O} 2)}$ changes dramatically in the transition to T1 phase.

The structure of the $\mathrm{Mn}_{3-x} \mathrm{Zn}_{x} \mathrm{O}_{4}$ spinels is decisively influenced by the presence of $\mathrm{Mn}^{3+}$. The content of $\mathrm{Mn}^{3+}$ in the sample depends on both, its composition and the temperature of its firing. The $\mathrm{Mn}^{3+}$ ion causes deformation of $\mathrm{MnO}_{6}^{9-}$ octahedron due to The Jahn-Teller effect. With the mixed manganese oxidation state of $3.5+$, the deformations of the octahedra are already detected in the cubic $\mathrm{C}$ phase, which is stable at low temperatures at the composition with $x=1.67$. Only when the deformations are oriented to a common axis at temperatures close to $700^{\circ} \mathrm{C}$, the long-range structure is transformed into tetragonal T2. With increasing temperature, the content of $\mathrm{Mn}^{3+}$ increases and the value of $x$ has to be decreased to obtain the single-phase T2. In parallel, the tetragonal character of T2 phase increases. At $x=1$, all $\mathrm{Mn}$ is in the oxidation state 3+ and the tetragonal $\mathrm{T} 1$ phase is stable, independent of the temperature.

\section{Conclusions}

The structures of the spinel-type phases from the $\mathrm{Zn}-\mathrm{Mn}-\mathrm{O}$ system were studied using X-ray absorption techniques. For the analysis, single-phase samples are needed so accordingly, singlephase spinel samples with different $\mathrm{Mn}_{3-x} \mathrm{Zn}_{x} \mathrm{O}_{4}$ compositions were prepared with calcination of oxalate precursors at different temperatures.

In the cubic $\mathrm{C}$ phase, stable below $700^{\circ} \mathrm{C}$ for a fixed composition of $x=1.67$, XANES analysis showed average manganese valence of $3.5+\left(\mathrm{Mn}_{1.33}^{3.5+} \mathrm{Zn}_{1.67} \mathrm{O}_{4}\right)$. EXAFS showed deformations of the $\mathrm{MnO}_{6}$ octahedra due to the Jahn-Teller effect caused by $\mathrm{Mn}^{3+}$. In the $\mathrm{C}$ phase, the deformations of $\mathrm{MnO}_{6}$ octahedra are randomly oriented. Their orientation to a common $c$-axis results in the transformation into a tetragonal T2 phase. The $\mathrm{Mn}$ valence in this phase changes with temperature, so the composition of the single-phase samples also changes with temperature. Consequently, the temperature and the composition are coupled and their influences cannot be discussed separately in this phase: the tetragonal deformation of the T2 phase increases with increasing temperature and the concurrent decrease of $x$ as the content of $\mathrm{Mn}^{3+}$ increases. At $x=1$, the $\mathrm{Mn}$ valence is $3+$ and the composition is fixed at $\mathrm{ZnMn}_{2} \mathrm{O}_{4}$, independent of the temperature: tetragonal T1 phase.

Modeling of EXAFS spectra of the $\mathrm{C}$ and $\mathrm{T} 2$ phases with an $a b$ initio FEFF model suggested full occupation of tetrahedral lattice sites by $\mathrm{Zn}$ and occupation of octahedral sites by both cations. In the $\mathrm{T} 1$ phase, a low degree of inversion was detected.

\section{Acknowledgment}

Access to synchrotron radiation facilities of HASYLAB (beamline C) is acknowledged. We would like to thank Edmund Welter of HASYLAB for expert advice on beamline operation. M. P. also acknowledges the Secretaría de Estado de Universidades e Investigación del Ministerio de Ciencia e Innovación (Spain) for the financial support. 


\section{References}

${ }^{1}$ S. Guillemet-Fritsch, C. Chanel, J. Sarrias, S. Bayonne, A. Rousset, X. Alcobe, and M. L. M. Sarrion, "Structure, Thermal Stability and Electrical Properties of Zinc Manganites," Solid State Ionics, 128 [1-4] 233-42 (2000).

${ }^{2}$ G. Ferraris, G. Fierro, M. Lo Jacono, M. Inversi, and R. Dragone, "Catalytic Activity of Copper-Zinc Manganites for the Reduction of $\mathrm{NO}$ and $\mathrm{N}_{2} \mathrm{O}$ by $\mathrm{Hy}-$ drocarbons," Appl. Cat. B-Environ., 45 [2] 91-101 (2003).

${ }^{3}$ G. Fierro, R. Dragone, and G. Ferraris, "NO and $\mathrm{N}_{2} \mathrm{O}$ Decomposition and their Reduction by Hydrocarbons Over Fe-Zn Manganite Spinels," Appl. Cat. B-Environ., 78 [1-2] 183-91 (2008).

${ }^{4}$ G. Monros, J. Carda, M. A. Tena, P. Escribano, J. Badenes, and E Cordoncillo, "Spinels from Gelatine-Protected Gels," J. Mater. Chem., 5 [1] 85-90 (1995).

${ }^{5}$ T. Dietl and H. Ohno, "Zener Model Description of Ferromagnetism in ZincBlende Magnetic Semiconductors," Science, 287 [5455] 1019-22 (2000).

${ }^{6}$ S. A. Wolf, D. D. Awschalom, R. A. Burhman, J. M. Daughton, S. Von Molnar M. L. Roukes, A. Y. Chtchelkanova, and D. M. Treger, "Spintronics: A Spin-Based Electronics Vision for the Future," Science, 294 [5546] 1488-95 (2001).

${ }^{7} \mathrm{H}$. Toussaint, "Solid State Reactions of $\mathrm{MnO}_{2}$ and $\mathrm{Mn}_{2} \mathrm{O}_{3}$ with $\mathrm{CaO}, \mathrm{ZnO}$, MgO and CdO," Rev. Chim. Min., 1 [2] 141-95 (1964).

${ }^{8}$ F. C. M. Driessen and G. D. Rieck, "Phase Equilibria in the System Zn-Mn-O in Air," J. Inorg. Nucl. Chem., 28 [8] 1593-600 (1966).

${ }^{9}$ J. Blasco and J. García, "Stable Cubic Spinels in the $\mathrm{Zn}-\mathrm{Mn}-\mathrm{O}$ System in Air," J. Solid State Chem., 179 [7] 2199-205 (2006).

${ }^{10} \mathrm{M}$. Peiteado, A. C. Caballero, and D. Makovec, "Diffusion and Reactivity of $\mathrm{ZnO}-\mathrm{MnO}_{x}$ System," J. Solid State Chem., 180 [9] 2459-64 (2007).

${ }^{11}$ M. Peiteado, A. C. Caballero, and D. Makovec, "Phase Evolution of Zn1-xMn $x$ O System Synthesized Via Oxalate Precursors," J. Eur. Ceram. Soc., 27 [13-15] 3915-8 (2007).

${ }^{12}$ S. Mogck, B. J. Kooi, and J. Th. M. De Hosson, "Tailoring of Misfit Along Interfaces Between $\mathrm{Zn}_{x} \mathrm{Mn}_{3-x} \mathrm{O}_{4}$ and Ag," Acta Mater., 52 [20] 5845-51 (2004).

${ }^{13}$ M. Peiteado, S. Sturm, A. C. Caballero, and D. Makovec, " $\mathrm{Mn}_{3-x} \mathrm{Zn}_{x} \mathrm{O}_{4}$ Spinel Phases in the Zn-Mn-O System," Acta Mater., 56 [15] 4028-35 (2008).

${ }^{14}$ R. G. Zakharov and S. A. Petrova, "Equilibrium and Non-Equilibrium Cation Distribution in $\mathrm{Zn}_{-} \mathrm{Mn}_{3} \mathrm{O}_{4}$ "; pp. 352-6 in Materials Science Forum, Vol. 321-323, European Power Diffraction Part $1 \& 2$, Edited by R. Delhez, and E. J. Mittemeijer. Trans Tech Publications Ltd., Switzerland, 2000.
${ }^{15}$ G. Lawes, A. S. Risbud, A. P. Ramirez, and R. Seshadri, "Absence of Ferromagnetism in Co and Mn Substituted Polycrystalline ZnO," Phys. Rev. B, 71 [4] 045201, 5pp (2005)

${ }^{16} \mathrm{M}$. Bouloudenine, N. Viart, S. Colis, J. Kortus, and A. Dinia, "Antiferromagnetism in Bulk Zn1-xCoxO Magnetic Semiconductors Prepared by the Coprecipitation Technique," Appl. Phys. Lett., 87 [5] 052501, 3pp (2005).

${ }^{17}$ B. Ravel and M. Newville, "ATHENA, ARTEMIS, HEPHAESTUS: Data Analysis for X-Ray Absorption Spectroscopy using IFEFFIT,” J. Synchrotron Radiat., 12 [4] 537-41 (2005).

${ }^{18}$ J. J. Rehr, R. C. Albers, and S. I. Zabinsky, "High Order Multiple Scattering Calculations of X-Ray Absorption Fine Structure," Phys. Rev. Lett., 69 [23] 3397400 (1992).

${ }^{19} \mathrm{U}$. Konig and G. Chol, "X-Ray and Neutron Diffraction in Ferrites of $\mathrm{Mn}_{x} \mathrm{Zn}_{1-x} \mathrm{Fe}_{2} \mathrm{O}_{4}$ Type," J. Appl. Cryst., 1 [2] 124-6 (1968).

${ }^{20} \mathrm{~S}$. Calvin, E. E Carpenter, V. G. Harris, and S. A. Morrison, "Use of Multiple-Edge Refinement of X-Ray Absorption Fine Structure to Determine Site Occupancy in Mixed Ferrite Nanoparticles," Appl. Phys. Lett., 81 [20] 3828-30 (2002).

${ }^{21}$ J. Wong, F. W. Lytle, R. P. Messmer, and D. H. Maylotte, "K-Edge Absorption-Spectra of Selected Vanadium Compounds," Phys. Rev. B, 30 [10] 5596 610 (1984).

${ }^{22}$ N. Z. Logar, N. N. Tusar, G. Mali, M. Mazaj, I. Arcon, D. Arcon, A. Recnik, A. Ristic, and V. Kaucic, "Manganese-Modified Hexagonal Mesoporous Aluminophosphate MnHMA: Synthesis and Characterization," Micropor. Mesopor Mater., 96 [1-3] 386-95 (2006).

${ }^{23}$ T. Ressler, J. Wong, and J. Roos, "Manganese Speciation in Exhaust Particulates of Automobiles using MMT-Containing Gasoline," J. Synchrotron Radiat., 6 [3] 656-8 (1999).

${ }^{24}$ D. Makovec, A. Kodre, I. Arcon, and M. Drofenik, "Structure of Manganese Zinc Ferrite Spinel Nanoparticles Prepared with Co-Precipitation in Reversed Microemulsions," J. Nanopart. Res., 11 [5] 1145-58 (2009).

${ }^{25}$ H. S. C. O'Neill, "Temperature-Dependence of the Cation Distribution in Zinc Ferrite $\left(\mathrm{ZnFe}_{2} \mathrm{O}_{4}\right)$ from Powder XRD Structural Refinement," Eur. J. Mineral., 4 [3] 571-80 (1992).

${ }^{26}$ T. Sato, K. Haneda, M. Seki, and T. Iijima, "Morphology and Magnetic-Properties of Ultrafine $\mathrm{ZnFe}_{2} \mathrm{O}_{4}$ Particles," Appl. Phys. A, 50 [1] 13-6 (1990). 